\title{
Research problems in clothing simulation
}

\author{
Kwang-Jin Choi ${ }^{*}$, Hyeong-Seok Ko \\ Graphics and Media Lab, Seoul National University, Seoul, South Korea
}

\begin{abstract}
Clothing simulation and animation are of great importance in computer animation. If cloth simulations could be improved to the point that they could generate realistic cloth motion in real-time, they would find uses in many aspects of daily life such as in fashion design and manufacturing. The area of cloth simulation and animation is full of technical challenges: creating more realistic results, achieving faster runtimes, and developing methods capable of constructing and simulating more complex garments. This paper provides an overview of the key procedures involved in the creation of clothed characters, describes the current state-of-the-art techniques, and proposes the research problems that most require further study. Three technical aspects of cloth simulation are considered in this paper: garment construction, physically based simulation, and collision resolution.
\end{abstract}

(C) 2004 Elsevier Ltd. All rights reserved.

Keywords: Cloth simulation; Garment design; Collision resolution

\section{Introduction}

The ability to clothe animated characters in fashionable clothing would add another dimension of richness to 3D animation. Considering the high degree of detail in current character animations, it is easy to see that techniques that give realistic, stable, fast clothing animation will be in everincreasing demand.

Thanks to the pioneering work of various groups over the past decade [2,5-8,16,39], cloth can now be simulated with remarkable realism. For example, natural wrinkles can now be produced using the particle model with the immediate buckling assumption, and the robustness of the collision handling in cloth simulations has been considerably improved. Along with the improvements that have been made in animation quality, the overall simulation algorithm has been refined such that it runs at a reasonable speed.

Unfortunately, clothing simulation cannot produce the motion of cloth to a satisfactory level, even when the simulation is restricted to the garments constructed using only very basic fashioning features such as cutting and sewing.

\footnotetext{
* Corresponding author.

E-mail addresses: kjchoi@graphics.snu.ac.kr (K.-J. Choi), ko@ graphics.snu.ac.kr (H.-S. Ko).
}

The central position of clothing in human life means that clothing simulations have many current and potential applications. Moreover, developments in the simulation of clothing should impact the field of animation in general. Thus, it is worthwhile to stand back and contemplate the overall clothing simulation process so as to see where the difficulties lie and to identify research problems that need to be solved.

This paper presents a retrospective of past work and proposes research directions for further developments. Each of the subsequent sections corresponds to a subtask of the overall clothing simulation process. In each section, we describe the desired function of the task, the methods proposed so far to perform the task, and the problems that require further study.

\section{Garment construction}

The basic problem confronting garment designers is the nonintuitive task of clothing a 3D character with a garment constructed from 2D patterns. In fact, it takes years for a person to become an expert pattern maker; even the design of simple garments such as T-shirts and skirts calls for the expertise of an experienced pattern maker. In clothing simulation, the generation of garments that both have 
the required design and fit the animated character is a timeconsuming preprocess that most animators dislike. Although numerous pattern CAD packages are available, they are targeted at pattern makers and the non-expert will find few of them to be user-friendly. The necessity of engaging an expert pattern maker to create interesting garments is one of the factors hindering rich clothing animation. Thus, the development of more intuitive garment design techniques suitable for use by non-experts is an important challenge in cloth simulation.

\subsection{Previous work}

Recently, an online made-to-measure system was presented by Cordier et al. [10]. Their system allows interactive adjustment of the 3D mannequin according to the shopper's body measurements, online resizing of the garment to fit the mannequin, and real-time simulation of the garment corresponding to the body motion.

An alternative approach to the problem of constructing garments is designing garment directly in 3D space and flattening the designed $3 \mathrm{D}$ surfaces to $2 \mathrm{D}$ plane. The $2 \mathrm{D}$ patterns obtained from flattening procedure will produce the desired design when they are sewed along the seam lines generated during the flattening procedure. Wang et al. [43] presented a garment design system that allows users to design 3D garment around a 3D human model through 2D sketch strokes (Fig. 1(a)). They add seam lines (Fig. 1(b)), then flatten the resulting $3 \mathrm{D}$ patterns to obtain a set of $2 \mathrm{D}$ patterns that can be used for manufacturing the garment (Fig. 1(c) and (d)). In the 3D garment design procedure, the

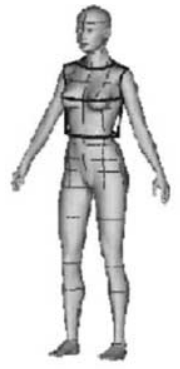

(a) input strokes to specify profile

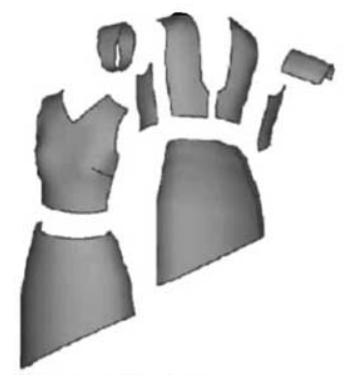

(c) separated 3D patterns
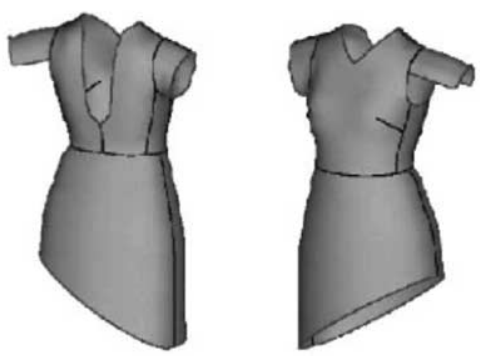

(b) constructed/edited surface \& painted seam lines

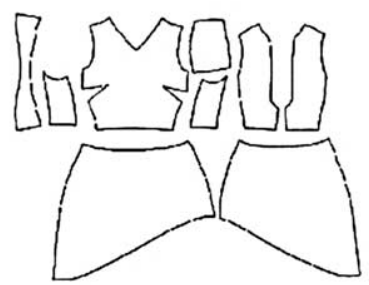

(d) flattend $2 \mathrm{D}$ patterns
Fig. 1. Overview of the garment design process in Wang et al.'s work [43] (courtesy of Charlie C.L. Wang). surface flattening technique plays a crucial role since it determines the quality of the final manufactured garments. Several flattening techniques have been proposed so far $[1,26,30,42]$. They are basically strain energy minimization processes though they differ in optimization technique, energy function formulation, etc.

\subsection{Research problems}

\subsubsection{Developing pattern generation techniques}

A difficulty that arises in garment construction is that patterns designed for one character cannot easily be adapted to differently sized characters. One approach to alleviating this problem would be to adopt the pattern grading methods used by pattern makers. However, discussion with skilled pattern designers reveals that (1) the grading procedure heavily depends on the specific design of a garment, and (2) there is no automatic or systematic way to modify a given pattern design to fit differently sized bodies. In practice, the grading procedure used in the fashion industry relies in large part on designers' experience, and therefore cannot be straightforwardly transferred to a computational procedure. Another, totally different approach to pattern generation would be to directly modify the 3D design of the garment and project the final result into $2 \mathrm{D}$ space to obtain the patterns. Such an approach should be more intuitive, given that the ultimate aim is to design a three-dimensional garment. However, this approach has yet to be developed to a practically useful level. The development of an automatic, intuitive pattern generation method remains a research challenge that, when solved, will have a dramatic impact on the fashion industry as well as on character animation.

\section{Physically based simulation}

Cloth modeling research began in the textile engineering community in the 1930s. Research in this area received a boost in the mid-1980s, when the computer graphics community started to study cloth modeling and animation techniques. One of the major concerns of the textile community is the accurate modeling and prediction of the highly non-linear behavior of cloth. In contrast, computer graphics researchers have tended to focus on efficiently reproducing the appearance of cloth. In computer graphics research, efficiency, stability, and visual realism are more important than accuracy. To achieve those characteristics, numerous simulation techniques have been proposed in the graphics literature.

\subsection{Previous work}

In the field of computer animation, Terzopoulos et al. [35] were the first to develop a physical model for use in the simulation of cloth, and Carignan et al. [7] refined this model by adding damping and collision-handling features. 
Breen et al. [5] developed a non-continuum particle model for predicting cloth drape. Later, Eberhardt et al. [16] adapted the model of Breen et al. to create a dynamic simulation method based on a Lagrangian formulation. Volino et al. [39] developed a cloth model based on elasticity theory and used a Newtonian formulation instead of a Lagrangian formulation. Eischen et al. [17] modeled cloth using non-linear shell theory. In their formulation, a standard nonlinear finite element procedure was used to obtain the system equation.

Another vital step in the generation of cloth motion is the numerical simulation of the underlying physical model. Since Baraff and Witkin [2] started using the semi-implicit method, the technique has become of a popular technique for integrating the equations of motion in cloth simulation. This method has been rigorously analyzed by various groups [20, 37,38]. Desbrun et al. [13] precomputed the inverse of the simplified Hessian matrix to make possible real-time cloth simulation. Kang et al. [24] proposed another variation of the semi-implicit method that avoids both solving the large linear system and precomputing the inverted Hessian matrix.

The abovementioned physical models were found to give quite realistic cloth motion; however Choi and Ko [8] revealed that those models suffer from a post-buckling instability that can be particularly problematic when wrinkles are formed. This instability is an inherent physical instability and is therefore independent of the numerical method employed. Noting that the buckling behavior of cloth differs from that of other thin materials, Choi and Ko assumed that application of a compressive force on cloth immediately initiates buckling rather than compression. Simulations incorporating this immediate buckling assumption produced realistic wrinkles without the post-buckling instability. Snapshots in Fig. 2 are taken from their simulation.

\subsection{Research problems}

The two key areas in which clothing simulations require improvement are the realism of the simulated cloth movement and the algorithm speed. Below we consider several research problems that are crucial to achieving the desired realism and speed.

\subsubsection{Modeling non-linear and hysteretic properties}

Current cloth simulations generate motions more characteristic of a rubbery material than of a cloth. This shortcoming arises because most cloth simulation techniques model the fabric as a linear elastic material. An obvious but non-trivial way to fix this problem would be to adopt a non-linear model that better captures the behavior of real fabrics. One difficulty that is likely to arise in any attempt to implement such a non-linear model is establishing the non-linear relationship between the stress and strain to a sufficient level of accuracy. Another anticipated problem of using a non-linear model is that the introduction of the non-linearity may increase the computational cost. Simple non-linear modeling may not be sufficient to recreate the behavior of a real fabric; for example, the non-linear stress-strain relationship in fabrics is known to follow different paths depending on whether the cloth is folding or unfolding. Hysteretic responses of this type are an important feature of fabric behavior that distinguish fabrics from other sheet materials. Several attempts have been made to incorporate hysteresis into models of fabric bending [16]. However, this property has been simulated only to a limited level, and methods for capturing the hysteretic nature of cloth during dynamic motion have yet to be developed.

\subsubsection{Exploring the continuum approach}

Currently, most cloth simulations use interacting particle models. An alternative to this approach is to model the cloth as a continuum. The finite element procedure, which is commonly used to analyze structural problems in solid continua, has several advantages over the interacting particle approach. For example, the continuum formulation is largely parameterization independent, even though regularly shape-and-sized discretization is required
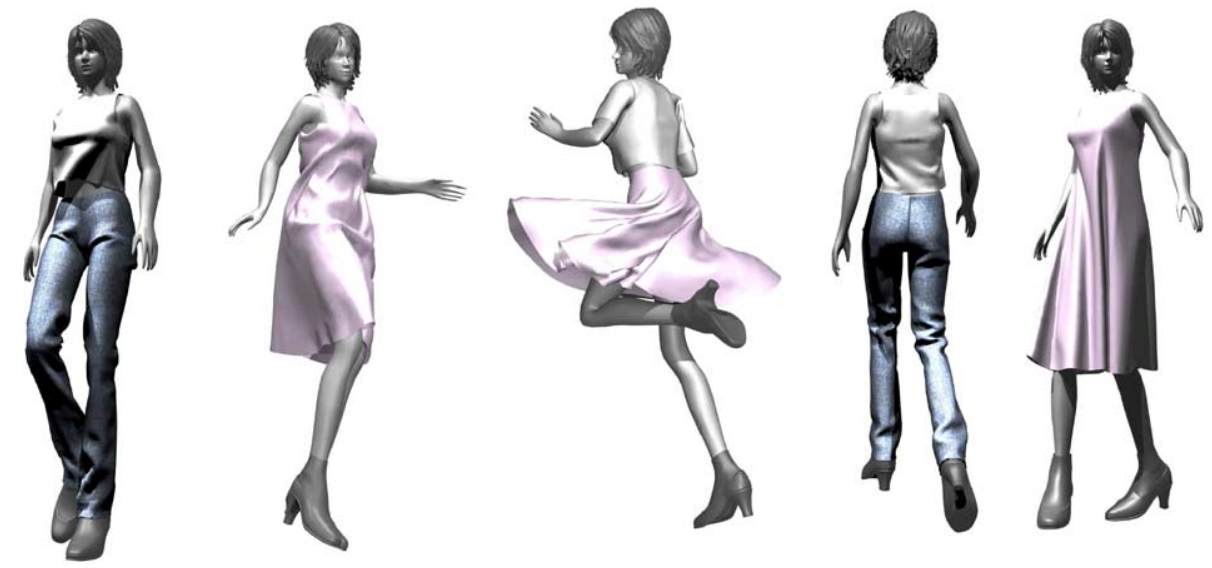

Fig. 2. Snapshots from Choi and Ko's work [8]. 
for better accuracy. This feature is important for practical reasons, because cloth simulators should be able to handle arbitrarily shaped, unstructured meshes representing cloth patterns. An additional attraction of the finite element approach is the large literature on the use of this framework for handling material and geometrical nonlinearity of structures. Although the finite element procedure provides technically sound and versatile tools, a number of complexities must be unraveled if this technique is to be used for simulating cloth. These include: (1) there is no straightforward and efficient way to alleviate the buckling instability problem, (2) the finite element procedure needs many more numerical operations than the particle formulation, and (3) collision resolution becomes a non-trivial task in the finite element formulation. At present, it is unclear whether the continuum approach gives better results, but we believe that this approach should be explored to test its viability.

\subsubsection{Increasing the fidelity of simulated cloth}

The ultimate aim of cloth simulation is to produce a simulated cloth that is indistinguishable from the real cloth being modeled. Judging this similarity on a frame-by-frame basis would be impractical. A more feasible alternative is to have experts assess the simulated fabric movements; if the experts find that the simulated fabric behaves exactly like the real fabric we may regard the simulation as a success. To achieve realistic modeling of a particular cloth, a reliable technique is required that can accurately extract the physical properties of the cloth and apply those properties to the simulated cloth. Currently, the Kawabata evaluation system (KES) is widely used to obtain the moment-curvature or force-shear relationship during cloth deformation.

An alternative to the use of KES measurements would be to identify a concise number of steerable control parameters that completely describe the static and dynamic material properties of a cloth. Currently, the final feel of simulated cloths is varied by adjusting the stiffness (in bending, stretching, and shearing), damping, air-drag, and mass. In [3], Bhat et al. estimated the above simulation parameters of a fabric by comparing the dynamic motion of the simulated fabric with that of the real fabric recorded with video cameras. Another complementary direction of research in addition to Bhat et al.'s work is to investigate whether there exist other parameters (e.g. moisture level, amount of hysteresis) that, if controlled in a simulation, increase the fidelity of the simulated cloth.

\subsubsection{Increasing algorithm speed while maintaining reasonable quality}

Computational efficiency is another key issue in cloth simulation. The speed of a simulation can be increased by employing a simplified physical model or a simplified numerical procedure. In regard to the physical model, there seems no meaningful simplification than the linear spring approximation in current use. On the other hand, several methods have been proposed for accelerating the numerical procedures, most of which are related to simplifying the implicit methods. However, all the simplifications proposed to date lead to a non-negligible degradation of the visual quality. Thus, the challenge is to reduce the amount of numerical computation in the implicit methods while maintaining quality at an acceptable level.

Any simplification of the implicit methods is expected to degrade the quality to some extent. However, by exploiting the fact that every part of the cloth does not need to be simulated to the same level of detail, we may be able to simplify selected aspects of the calculation without significantly degrading the quality. For example, we could apply an adaptive multi-resolutional approach such as that attempted by $[12,19]$. Currently, such methods are hampered by discontinuities at the transitions between different levels of detail. However, further work aimed at eliminating these artifacts could potentially give rise to a powerful technique capable of high-quality real-time cloth simulation.

The adaptive multi-resolutional approach uses higher resolution meshes for the portions of the cloth that undergo large deformations and/or the portions that are close to the camera. Further simplifications can potentially be made by noting that in some circumstances it is unnecessary to fully simulate the entire cloth. For example, the movement of tight clothes is highly dependent on the body motion, and therefore such cloth may not need full 3D simulation. Cordiner and Magnenat-Thalmann [11] were the first to propose a real-time simulation technique based on this idea. We expect that this type of simplification will be exploited in future studies to increase simulation speed.

The simulation speed can also be increased by utilizing the modern graphics architecture. The current GPU techniques $[4,21,28,29]$ allow the numerical operations or procedures frequently used in physically based simulation to be performed much faster than on CPU. To date, there has been no report on cloth simulation that exploited the new technology. The new hardware technology, if combined with further optimizations in simulation algorithms, may practically enable real-time clothing simulation.

\section{Collision resolution}

Collision resolution, which consists of detecting collisions and generating suitable responses, is one of the most time-consuming operations in cloth simulations. The main challenges confronting attempts to develop superior collision resolution techniques are reducing the number of comparisons by exploiting the temporal and spatial coherency, increasing the accuracy of collision detection, and creating physically correct and stable collision responses. 

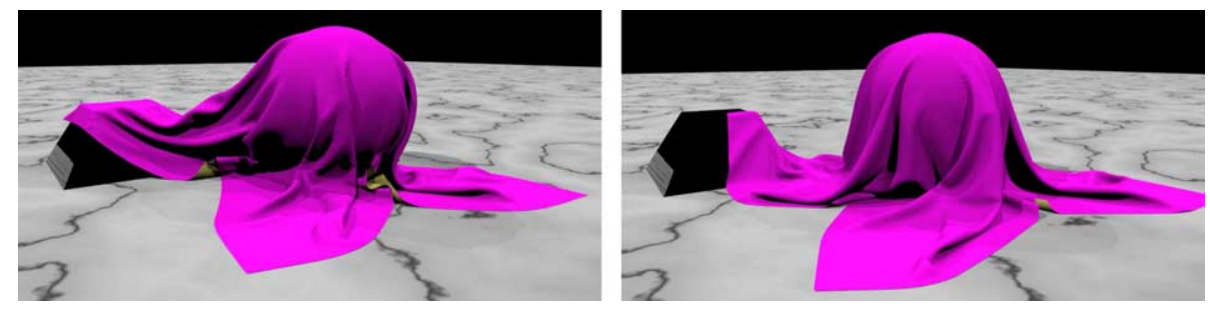

Fig. 3. Snapshots from Bridson et al.'s work [6] (courtesy of Robert Bridson).

\subsection{Previous work}

Several techniques have been developed to reduce the number of comparisons that must be made when detecting collisions in meshes consisting of many triangles. One approach is the use of bounding volume hierarchies $[18,27]$, which has been shown to significantly reduce the number of comparisons. Another popular method for reducing the number of comparisons is space subdivision. For example, Moore and Wilhelms [31] and Smith et al. [34] used octree structures, and Turk [36] used uniform subdivision. Zhang and Yuen [44] also developed a spatial enumeration method for collision detection. Their experimental data showed that the time complexity of their algorithm is close to $O(n)$.

To accurately detect the collisions of moving objects, intersection should be established in a four-dimensional space (i.e. three space coordinates and time). Hubbard [22] proposed a time critical detection algorithm based on testing for the intersection of four-dimensional hypertrapezoids. Duff [15] used interval analysis to quickly identify and refine the regions of the 4D space that can contain collisions. Lin et al. [9] used a similar 4D analysis that included an efficient technique for tracking the distance between pairs of convex polyhedra. Karabassi et al. [25] and Raab [33] used a swept volume approach which can find whether there are occurrences of collision during the given time interval more easily.

Volino and Thalmann [40] proposed a technique that increases the speed of self-collision detection by efficiently pruning the comparison pairs based on an analysis of surface curvature coherency. Subsequently, the same authors [41] presented a collision response model based on a geometric correction method that can robustly resolve multiple collisions. In this method, barycentric coordinates are used to calculate continuous collision responses among colliding geometric primitives such as vertices, edges, or triangles. In contrast, Provot [32] addressed the multiple collision problem by introducing the concept of a zone of impact. In this approach, the particles involved in a multiple collision are grouped together and the resulting group of particles is considered as a rigid object in the calculation of the collision response. Huh et al. [23] also proposed a method for handling multiple collisions based on the concept of a zone of impact. They divided the particles in an impact zone into collision clusters and then simultaneously resolved the collisions of those clusters to avoid possible subsequent collisions. Bridson et al. [6] proposed a robust collision handling technique that combines repulsive forces, geometric treatment of collisions, and rigid impact zones. The combination of those techniques made the collision resolution process run very efficiently. They additionally presented a subdivision scheme that avoids collisions during the subdivision steps. See Fig. 3 for the simulation results of Brdison et al.'s work.

\subsection{Research problems}

The factors that complicate the collision handling procedure are as follows.

\subsubsection{Rapid collision detection}

Cloths are thin and highly flexible materials that can undergo very large deformations. As a result, cloth motion involves many more collisions than the motion of volumetric objects. Given that the meshes used in cloth models commonly contain up to tens of thousands of triangles, collision detection is an enormous computational burden that must be carried out very efficiently. In collision detection algorithms, the most frequent operation is the pair-processing procedure that determines whether two objects have collided. Therefore, devising methods to prune the number of pairs is an area worthy of study. One approach to reducing the number of pairs is to use hierarchical bounding volumes; however this scheme may not be useful for highly deformable objects such as cloths because the hierarchy would need to be updated at every time step. An alternative approach that has received some attention $[11,40]$ is to prune the pairs by considering cloth-specific geometric conditions such as the curvature of the surface, or the movement of the human body over which the cloth is spread. This approach has shown some promise, but requires further study.

\subsubsection{Accurate collision detection}

Due to the thin structure of cloth, simple 3D intersection detection algorithms fail to capture some collisions even when a cloth is moving slowly. If a collision goes undetected, it is difficult to subsequently recover the valid state because the cloth surface has no outside/inside distinction as far as collision detection is concerned. Therefore, it is of the utmost importance that the collision detection algorithm detects all 
collisions. For undetected collisions, numerical error is often the source of the problem. For example, when solving the 4-point collision equation that is used to detect linearly approximated moving collisions between triangle/vertex or edge/edge [14,32], the resulting polynomials are frequently ill-conditioned and thus it is hard to detect the collisions represented by those polynomials. The erroneous detection of a collision when no collision has occurred does not give rise to problems provided the collision response does not lead to complex subsequent collisions. In contrast, missing a real collision ruins the remainder of the simulation. Thus, although the accuracy of the internal dynamics can be sacrificed to enhance simulation performance, inaccurate collision resolution results in catastrophe. Currently, collision detection failure is one of the main factors hindering the dynamic simulation of complex garments. Development of an accurate, bullet-proof collision detection algorithm would be a significant step forward for the field of cloth simulation.

\subsubsection{Robust collision response generation}

Once a collision is detected, the consequences of that collision must be simulated to generate the correct subsequent cloth movement. This process, referred to as collision response generation, has yet to be modeled in a completely satisfactory manner.

One of the thorniest problems in generating cloth collision responses is that resolving one collision often causes new secondary collisions. As a result of this characteristic, the system may not reach a collision-free state after application of a single resolution step. Thus, for a collision resolution method to be robust, it seems unavoidable that the result of a resolution step should be tested iteratively until no further collisions are detected.

The iterative approach outlined above, although correct from a theoretical viewpoint, has a serious potential drawback: the iterative process may take an impractical amount of time to arrive at a collision-free configuration. To date this problem has typically been circumvented by including a fail-safe action that enforces a collision-free state after a predetermined number of iterations. This fail-safe action consists of grouping the particles involved in the multiple collisions and then treating the resulting cluster as a rigid body. However, handling a portion of the simulated cloth as a rigid body introduces artifacts into the system. These artifacts range from a simple degradation of the verisimilitude of the cloth motion to the more serious problem that, due to the creation of rigid clusters, the cloth cannot pass through constricted spaces that the real cloth would be able to pass through.

For a collision resolution method to be of practical use, it must involve as few iterative steps as possible. Although fail-safe action achieves this aim, it does so at the cost of introducing potentially severe artifacts into the simulation. Thus, one of the key challenges in the field of cloth simulation is to develop a collision resolution technique that can find a collision free configuration with smaller amount of (or no) backtracking or fail-safe actions.

\section{Conclusion}

In this article we have presented an overview of the challenges confronting researchers in the field of cloth simulation. The ability to produce natural-looking cloth animations in real-time would be of great utility, especially considering the many contexts in which the human body is covered with cloth. The impact of clothing simulation is not limited to the field of computer animation. As the simulated cloth motion comes closer to the behavior of real cloth, the technique is expected to make a significant contribution to other areas of everyday life such as fashion design and textile engineering.

The eventual goals are to develop simulation techniques that (1) create more realistic cloth behavior, (2) run faster, and (3) are capable of constructing and simulating more complex garments. In this paper, we reviewed the current state-of-the-art in this area from three technical perspectives-garment construction, physically based simulation, and collision handling - and identified areas that require further study.

The list of challenges described here is not meant to be complete. Nevertheless, we have attempted to delineate the core challenges in the hope that an airing of these issues will stimulate researchers in related fields, and eventually contribute to achieving the aforementioned vision.

\section{Acknowledgements}

This work was supported by Korea Ministry of Information and Communication. This work was also partially supported by Automation and Systems Research Institute at Seoul National University, and the Brain Korea 21 Project.

\section{References}

[1] Azariadis PN, Aspragathos NA. Geodesic curvature preservation in surface flattening through constrained global optimization. Comput Aided Des 2001;33(8):561-617.

[2] David Baraff, Andrew Witkin. Large steps in cloth simulation. Proceedings of SIGGRAPH 98, computer graphics proceedings, annual conference series.: ACM, ACM Press/ACM SIGGRAPH; 1998 p. 43-54.

[3] Kiran Bhat, Christopher Twigg, Jessica Hodgins, Pradeep Khosla, Zoran Popović, Steven Seitz. Estimating cloth simulation parameters from video. ACM Symp Comput Animat 2003.

[4] Jeff Bolz, Ian Farmer, Eitan Grinspun, Peter Schröoder. Sparse matrix solvers on the gpu: conjugate gradients and multigrid. SIGGRAPH 2003 conference proceedings, annual conference series.: ACM Press/ACM SIG-GRAPH; 2003. 
[5] Breen David E, House Donald H, Wozny Michael J. Predicting the drape of woven cloth using interacting particles. Proceedings of SIGGRAPH 94, computer graphics proceedings, annual conference series.: ACM Press/ACM SIGGRAPH; 1994 p. 365-372.

[6] Robert Bridson, Fedkiw Ronald P, John Anderson. Robust treatment of collisions, contact, and friction for cloth animation. SIGGRAPH 2002 conference proceedings, annual conference series.: ACM Press/ACM SIGGRAPH; 2002 p. 594-603.

[7] Michel Carignan, Ying Yang, Nadia Magnenat-Thalmann, Daniel Thal-mann. Dressing animated synthetic actors with complex deformable clothes. Comput Graph (Proc ACM SIGGRAPH 92): ACM; 1992 p. 99-104.

[8] Kwang-Jin Choi, Hyeong-Seok Ko. Stable but resposive cloth. SIGGRAPH 2002 conference proceedings, annual conference series.: ACM Press/ACM SIGGRAPH; 2002 p. 604-611.

[9] Ming C. Lin, Dinesh Manocha, John Canny. Fast collision detection between geometric models. Technical Report TR93-004, Department of computer science, University of North Carolina at Chapel Hill; 1993.

[10] Frederic Cordier, Hyewon Seo, Nadia Magnenat Thalmann. Made-tomeasure technologies for an online clothing store. IEEE Comput Graph Appl 2003;23(1):38-48.

[11] Frederic Cordiner, Nadia Magnenat-Thalmann. Real-time animation of dressed virtual humans. Eurographics 2002.

[12] Gilles Debunne, Mathieu Desbrun, Marie-Paule Cani, Barr Alan H. Dynamic real-time deformations using space \& time adaptive sampling. SIG-GRAPH 2001, computer graphics proceedings.: ACM Press/ACM SIGGRAPH; 2001 p. 31-36.

[13] Mathieu Desbrun, Peter Schröder, Alan Barr. Interactive animation of structured deformable objects. Graph Interf 1999 p. 1-8.

[14] Doghri I, Muller A, Taylor RL. A general three-dimensional contact procedure for finite element codes. Eng Comput 1998;15(2):233-59.

[15] Tom Duff. Interval arithmetic and recursive subdivision for implicit functions and constructive solid geometry. SIGGRAPH'92: 19th international conference on computer graphics and interactive techniques, Chicago, Illinois, July 26-31.: ACM Press; 1992 p. 131-138.

[16] Bernhard Eberhardt, Andreas Weber, Wolfgang Strasser. A fast, flexible, particle-system model for cloth draping. IEEE Comput Graph Appl 1996;16(5):52-9.

[17] Eischen Jeffrey W, Shigan Deng, Clapp Timothy G. Finite-element modeling and control of flexible fabric parts. IEEE Comput Graph Appl 1996;16(5):71-80.

[18] Stefan Gottschalk, Ming Lin, Dinesh Manocha. OBB-Tree: a hierarchical structure for rapid interference detection. SIGGRAPH 96 conference proceedings, annual conference series, held in New Orleans, Louisiana, 04-09 August.: ACM SIGGRAPH, Addison Wesley; 1996 p. 171-180.

[19] Eitan Grinspun, Petr Krysl, Peter Schröder. Charms: A simple framework for adaptive simulation. SIGGRAPH 2002 conference proceedings, annual conference series.: ACM Press/ACM SIGGRAPH; 2002 p. 281-290.

[20] Hauth M, Etzmuß O. A high performance solver for the animation of deformable objects using advanced numerical methods. In: Chalmers A, Rhyne T-M, editors. Proceedings of Eurographics. Computer Graphics Forum, vol. 20(3) p. 319-328.

[21] Hillesland Karl E, Sergey Molinov, Radek Grzeszczuk. Nonlinear optimization framework for image-based modeling on programmable graphics hardware. SIGGRAPH 2003 conference proceedings, annual conference series.: ACM Press/ACM SIGGRAPH; 2003.

[22] Hubbard Philip M. Collision detection for interactive graphics applications. IEEE Trans Vis Comput Graph 1995;1(3):218-30.

[23] Suejung Huh, Dimitris Metaxas, Norman Badler. Collision resolutions in cloth simulation. Comput Animat 2001.

[24] Young-Min Kang, Jeong-Hyeon Choi, Hwan-Gue Cho, Chan-Jong Park. Fast and stable animation of cloth with an approximated implicit method. Comput Graph Int 2000 p. 247-256.
[25] Evaggelia-Aggeliki Karabassi, Georgios Papaioannou, Theoharis Theoharis. Intersection test for collision detection in particle systems. J Graph Tools: JGT 1999;4(1):25-37.

[26] Sung Min Kim, Tae Jin Kang. Garment pattern generation from body scan data. Comput Aided Des 2003;35(7):611-8.

[27] Klosowski James T, Mitchell Joseph SB, Henry Sowizral, Karel Zikan. Efficient collision detection using bounding volume hierarchies of k-DOPs. IEEE Trans Vis Comput Graph 1998;4(1):21-36.

[28] Jens Krueger, Ruediger Westermann. Linear algebra operators for gpu implementation of numerical algorithms. SIGGRAPH 2003 conference proceedings, annual conference series.: ACM Press/ACM SIGGRAPH; 2003.

[29] Mark William R, Steven Glanville R, Kurt Akeley, Kilgard Mark J. $\mathrm{Cg}$ : a system for programming graphics hardware in a c-like language. SIGGRAPH 2003 conference proceedings, annual conference series.: ACM Press/ACM SIGGRAPH; 2003.

[30] McCartney J, Hinds BK, Seow BL. The flattening of triangulated surfaces incorporating darts and gussets. Comput Aided Des 1999; 31(4):237-97.

[31] Matthew Moore, Jane Wilhelms. Collision detection and response for computer animation. Comput Graph (SIGGRAPH'88 Proc) 1988 p. 289-298.

[32] Xavier Provot. Collision and self-collision handling in cloth model dedicated to design garments. Graph Interf'97 1997 p. $147-154$

[33] Sigal Raab. Controlled perturbation for arrangements of polyhedral surfaces with application to swept volumes. Proceedings of the conference on computational geometry (SCG'99), New York, NY, June 13-16.: ACM Press; 1999 p. 163-172.

[34] Smith A, Kitamura Y, Takemura H, Kishino F. A simple and efficient method for accurate collision detection among deformable objects in arbitrary motion. Proceedings of the IEEE virtual reality annual international symposium 1995 p. 136-145.

[35] Demetri Terzopoulos, John Platt, Alan Barr, Kurt Fleischer. Elastically deformable models. Proceedings of the 14th annual conference on computer graphics and interactive techniques.: ACM Press; 1987 p. 205-214.

[36] Greg Turk. Interactive collision detection for molecular graphics. Master's thesis. Department of Computer Science, University of North Carolina at Chapel Hill; 1989.

[37] Volino P, Magnenat-Thalmann N. Implementing fast cloth simulation with collision response. Proceedings of the conference on computer graphics international (CGI-00); June 19-24 2000 p. 257-268.

[38] Volino P, Magnenat-Thalmann N. Comparing efficiency of integration methods for cloth animation. Proceedings of the conference on computer graphics international (CGI-01) 2001.

[39] Pascal Volino, Martin Courshesnes, Nadia Magnenat Thalmann. Versatile and efficient techniques for simulating cloth and other deformable objects. Proceedings of SIGGRAPH 95, computer graphics proceedings, annual conference series.: ACM Press/ACM SIG-GRAPH; 1995 p. 137-144.

[40] Pascal Volino, Nadia Magnenat Thalmann. Collision and selfcollision detection: Efficient and robust solutions for highly deformable surfaces. Computer animation and simulation'95, eurographics.: Springer-Verlag; 19953-211-82738-2 p. 55-65.

[41] Pascal Volino, Nadia Magnenat Thalmann. Accurate collision response on polygonal meshes. Comput Animat 2000.

[42] Wang Charlie CL, Smith Shana S-F, Yuen Matthew MF. Surface flattening based on energy model. Comput Aided Des 1999;34(11): 769-868.

[43] Wang Charlie CL, Yu Wang, Yuen Matthew MF. Feature based 3d garment design through 2d sketches. Comput Aided Des 2003;35(7): 659-72.

[44] Zhang D, Yuen M. Collision detection for clothed human animation. Proceedings of the 8th Pacific graphics conference on computer graphics and application (PACIFIC GRAPHICS-00); October 3-5 2000 p. $328-337$. 


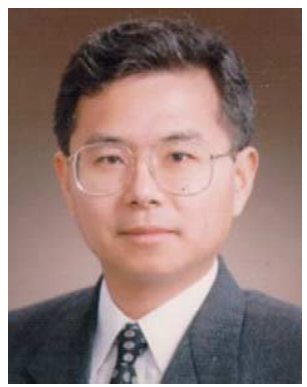

Hyeong-Seok Ko is an associate professor in the School of Electrical Engineering at Seoul National University. Currently, he is the Director of Graphics and Media Lab at the same university, and is actively developing the techniques for realizing Digital Actors, the actors created by computer graphics technology which are so real that people cannot tell if they are animated or captured from the real world. Before joining Seoul National University, he has held an assistant professor position at the University of Iowa. Ko received B.A. and M.S. degrees in Computer Science from Seoul National University in 1981 and 1985. He received a Ph.D. degree in Computer and Information Science from University of Pennsylvania. $\mathrm{He}$ is interested in physics-based simulations including clothing simulation, hair modeling and simulation, fluid simulation, simulation of dynamic deformation, and physically-based retargeting of humanoid motions.

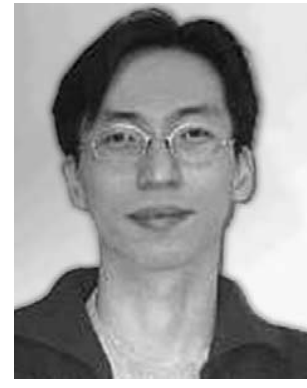

Kwang-Jin Choi is a postdoctoral associate in Graphics and Media Lab in Seoul National University. He received $\mathrm{BS}$, MS and $\mathrm{PhD}$ degrees in Electrical Engineering from Seoul National University in 1996, 1998, and 2003. $\mathrm{He}$ is interested in physics-based simulation and special effects. 